\title{
FLORÍSTICA E FITOSSOCIOLOGIA DE ESPÉCIES ARBÓREAS E ARBUSTIVAS EM UMA ÁREA DE CAATINGA EM ARCOVERDE, PE, BRASIL ${ }^{1}$
}

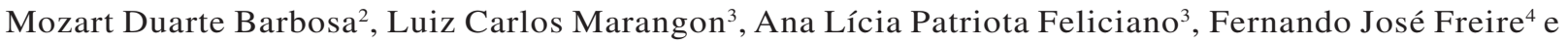
Gildete Maria Tenório Duarte ${ }^{5}$

\begin{abstract}
RESUMO - Os estudos de florística e fitossociologia contribuem significativamente para o conhecimento das formações florestais, visto que evidenciam a riqueza e heterogeneidade dos ambientes avaliados. O trabalho foi realizado em um fragmento de Caatinga hipoxerófila no Município de Arcoverde, PE, e teve como objetivo verificar a composição florística e parâmetros fitossociológicos das espécies arbóreas e arbustivas que compõem o fragmento. O estudo foi realizado em 40 parcelas de 10 × $25 \mathrm{~m}$, sendo o nível de inclusão a circunferência a $1,3 \mathrm{~m}$ do solo com $\mathrm{CAP} \geq 10 \mathrm{~cm}$. Foram estimados a densidade absoluta e relativa, a frequência absoluta e relativa, a dominância absoluta e relativa e o valor de importância; a diversidade específica foi dada pelo índice de diversidade de Shannon-Weaver (H') e equabilidade de Pielou, sendo determinada a distribuição hipsométrica em intervalos de $1 \mathrm{~m}$ e distribuição diamétrica em intervalos com amplitude de $3 \mathrm{~cm}$. No levantamento florístico das adultas, foram encontrados 1.491 indivíduos vivos, distribuídos em 36 espécies arbóreo-arbustivas, 19 famílias e 31 gêneros. As famílias que mais se destacaram foram Mimosaceae, Euphorbiaceae, Anacardiaceae, Caesalpiniaceae e Rhamnaceae. O índice de diversidade de Shannon-Weaver foi de 2,05 nats/ind. e a equabilidade de Pielou, de 0,57. Nos sete parâmetros fitossociólogicos avaliados, as espécies que mais se destacaram foram Mimosa ophthalmocentra, Poincianella pyramidalis, Senegalia bahiensis, Senegalia paniculata, Croton blanchetianus e Mimosa tenuiflora, indicando ser essas espécies as mais bem adaptadas ao ambiente avaliado, tanto nas condições edafoclimáticas quanto na competição com as demais espécies presentes no fragmento de Caatinga em Arcoverde, PE.
\end{abstract}

Palavras-chave: Fragmento, Diversidade e Estrutura.

\section{FLORISTIC AND PHYTOSOCIOLOGY OF TREE AND SHRUB SPECIES IN CAATINGA AREA IN ARCOVERDE, PERNAMBUCO, BRAZIL}

\begin{abstract}
The floristic and phytosociological studies contribute significantly to the knowledge of forest formations by showing the richness and diversity of the environments evaluated. This work was performed in a hypoxerophytic Caating a fragment in the city of Arcoverde, Pernambuco state and aimed at determining the floristic composition and phytosociological parameters of tree and shrub species that make up the fragment. The study was conducted in 40 plots $(10 \times 25 \mathrm{~m})$ with inclusion level the circumference at $1.3 \mathrm{~m}$ above the ground with $C A P \geq 10 \mathrm{~cm}$. The absolute and relative density, absolute and relative frequency, absolute and relative dominance and importance value were evaluated; species diversity was given by index of ShannonWeaver $\left(H^{\prime}\right)$ and equality of Pielou evenness, determining the hypsometric distribution at one meter intervals and diameter distribution at $3 \mathrm{~cm}$ intervals. In the floristic survey of adults, 1,491 individuals were found alive, distributed in 36 woody tree and shrub species, 19 families and 31 genera. Families that stood out most were Mimosaceae, Euphorbiaceae, Anacardiaceae, Caesalpiniaceae and Rhamnaceae. The diversity index of Shannon-Weaver was 2.05 nats/ind. and equality of Pielou evenness was 0.57 . For the seven phytosociological parameters evaluated, the species that stood out were Mimosa ophthalmocentra, Poincianella pyramidalis,
\end{abstract}

\footnotetext{
${ }^{1}$ Recebido em 20.03.2012 aceito para publicação em 04.06.2012.

${ }_{2}^{2}$ Departamento de Biologia e Geografia, Centro de Ensino Superior de Arcoverde/AESA-CESA, Brasil, E-mail:<barbosamd@ig.com.br>.

${ }^{3}$ Departamento de Ciência Florestal, Universidade Federal Rural de Pernambuco/UFRPE, Brasil, E-mail: <marangon@ dcfl.ufrpe.br> e $<$ licia@dcfl.ufrpe.br>.

${ }^{4}$ Departamento de Agronomia, Universidade Federal Rural de Pernambuco/UFRPE, Brasil, E-mail: <f.freire@ depa.ufrpe.br>.

${ }^{5}$ Secretaria de Educação de Pernambuco - Arcoverde/SEDUC, E-mail: <mariagiltd @ig.com.br>.
} 
Senegalia bahiensis, Senegalia paniculata, Croton blanchetianus and Mimosa tenuiflora, indicating that these are the species best adapted to the environment assessed, both in environmental conditions and competition with other species present in the fragment of Caatinga in Arcoverde-PE.

Keywords: Diversity, Frament and Structure.

\section{INTRODUÇÃO}

Para Andrade-Lima (1981), a caatinga, em geral, é caracterizada como formação arbóreo-arbustiva restrita ao domínio do clima semiárido do Nordeste brasileiro. Essa vegetação tem sido classificada como savana estépica, hierarquizada em diversas tipologias (IBGE, 1991).

De acordo com Sampaio (1995), devido à grande extensão territorial, a caatinga encerra enorme variabilidade de faciações fitogeográficas evidenciadas, principalmente, pelas diferenças fisionômicas, densidades, composição de espécies e aspectos fenológicos. A cobertura vegetal é representada por formações xerófilas e caducifólias - a caatinga - muito diversificadas por razões climáticas, edáficas, topográficas e antrópicas. A caatinga apresenta certo número de problemas quanto à sua definição e classificação, em virtude de serem encontradas de maneira muito heterogênea não só do ponto de vista fisionômico, da sua composição florística e condições estacionais, assim como pelos mais de 500 anos de atividade humana centrada na pecuária, na agricultura, na exploração mineral e no extrativismo vegetal (ALVES, 2007). Apesar da significativa extensão, importância socioeconômica e ser o único bioma com ocorrência restrita ao território nacional, a Caatinga é o menos protegido dos biomas brasileiros, com menos de $2 \%$ de sua área estando sob a forma de unidades de conservação de proteção integral (TABARELLI et al., 2000).

A caatinga é uma das regiões menos conhecidas da América do Sul, no que diz respeito à sua biodiversidade (SILVA; TABARELLI, 1999). Apesar do conhecimento insuficiente, a caatinga vem sofrendo alterações drásticas na sua estrutura (BRASIL, 1999). O bioma Caatinga vem sendo sistematicamente devastado, já que há muitos séculos o homem vem usando a área recoberta pela caatinga com pecuária intensiva, agricultura nas partes mais úmidas, retirada de lenha e madeira e outros fins de menor interesse socioeconômico. Esse tipo de exploração em um ambiente complexo poderá levá-lo a um processo irreversível de degradação (SANTANA; SOUTO, 2006). Segundo Andrade et al. (2005), o sistema agropastoril apresenta-se como o fator que maior pressão exerce sobre a cobertura vegetal do semiárido nordestino, e essa pressão varia de intensidade em função da localização, estrutura e tamanho dos remanescentes.

Entre essas áreas de caatinga, Sá et al. (2003) indicaram a área denominada carvoejamento, abrangendo os Municípios de Arcoverde, Buique, Tupanatinga, Calumbi, Flores, Betânia, Serra Talhada, Ibimirim, Floresta, Sertânia e Custódia, onde existe inadequada exploração da vegetação nativa e deve ser considerada como uma das áreas prioritárias para conservação.

Nos últimos anos, diversos trabalhos fitossociológicos e florísticos foram realizados em áreas de caatinga (LEMOS; RODAL, 2002; PEREIRA et al., 2002; ALCOFORADO-FILHO et al., 2003; MARACAJÁ et al., 2003; CESTARO; SOARES, 2004; ANDRADE et al., 2005; LACERDA et al., 2005, PEGADO et al., 2006; QUEIROZ et al., 2006; SANTANA; SOUTO, 2006; FABRICANTE; ANDRADE, 2007; LACERDA et al., 2007; PINHEIRO; ALVES, 2007; PESSOA et al., 2008; RODAL et al., 2008a; RODAL et al., 2008b; SANTOS et al., 2008; RAMALHO et al., 2009; SOUZA; RODAL, 2010.), tendo fornecido informações sobre florística e estrutura da vegetação de caatinga.

Segundo Sampaio (1996), os estudos fitossociológicos fornecem informações acerca da estrutura das comunidades e de algumas populações, bem como o conhecimento da flora regional, subsidiando, dessa forma, o manejo, a recuperação e, ou, a conservação dos ecossistemas. Portanto, pode constituir o ponto de partida para nortear atividades conservacionistas.

Diante do exposto, este trabalho objetivou verificar a composição florística e fitossociológica das espécies arbóreo-arbustivas que compõem o fragmento de caatinga hipoxerófila de Arcoverde, PE.

\section{MATERIAL E MÉTODOS}

\subsection{Caracterização da área de estudo}

O Remanescente, fragmento de caatinga, pertence à fazenda Cavalcanti, localizada no povoado de Ipojuca, em Arcoverde, PE. Localiza-se a uma latitude de 
$08^{\circ} 01^{\prime} 03,6^{\prime \prime}$ sul e a uma longitude de $034^{\circ} 56^{\prime} 44,1^{\prime \prime}$ oeste, distante $22 \mathrm{~km}$ do distrito-sede, localizado na mesorregião Sertão e na Microrregião Sertão do Moxotó, do Estado de Pernambuco, na divisa entre o agreste e o sertão, distante $256 \mathrm{~km}$ do Recife (IBGE, 2008). A área total do remanescente era de 35 ha (Figura 1).

Esse fragmento está inserido no Planalto da Borborema, com altitude variando entre 650 e 1.000 $\mathrm{m}$, mais exatamente num planalto entre os contrafortes do Ororuba e as serras da Conceição, de Moça e de Porteira (CAVALCANTI, 2010). O solo do fragmento é classificado como Argissolo Vermelho-Amarelo.

O clima da região, segundo a classificação de Köppen, do tipo Bshs', é seco de estepe de baixa latitude, com chuvas de outono-inverno, temperaturas médias variando entre $12^{\circ} \mathrm{e} 25^{\circ} \mathrm{C}$ e precipitação pluviométrica média anual de $1.058,8 \mathrm{~mm}$ (IBGE, 2008). A precipitação foi de $1.037,5 \mathrm{~mm}$, com uma média de $86,46 \mathrm{~mm}$ no período de 2009 a 2010, em que o experimento foi avaliado.

A vegetação da área do fragmento é de caatinga hipoxerófila e há 51 anos não sofre corte raso ou queimada, entretanto tem sido utilizada para criação de bovinos em regime semiextensivo.

\subsection{Composição florística e estrutural}

O estudo florístico e estrutural foi realizado em 40 parcelas de 10 × 25 m (MARANGON et al., 2008), as quais obedeceram a uma distância entre transectos de $30 \mathrm{~m}$ e entre parcelas de $25 \mathrm{~m}$. O levantamento do componente arbóreo e arbustivo teve como nível de inclusão a circunferência do tronco a $1,30 \mathrm{~m}$ do solo, sendo a $\mathrm{CAP} \geq 10,0 \mathrm{~cm}$ e a altura, estimada. O material botânico fértil foi tambado no Herbário Professor

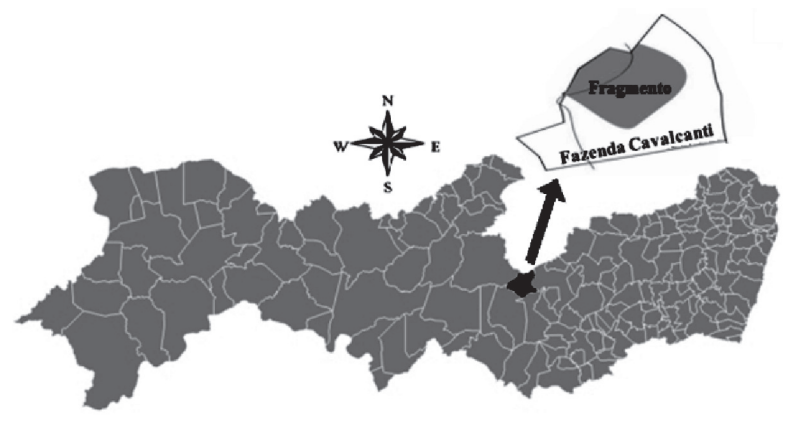

Figura 1 - Estado de Pernambuco com a localização do Município de Arcoverde, PE, e croqui do fragmento estudado.

Figure 1 -Pernambuco State with the location of the Municipality of Arcoverde-PE and sketch of the studied fragment.
Vasconcelos Sobrinho, do Departamento de Biologia da UFRPE, seguindo-se o sistema de classificação de Cronquist (1988).

Para análise dos dados dos indivíduos, foi utilizado o programa Mata Nativa 2 (CIENTEC, 2006), em que foram estimados os seguintes parâmetros fitossociológicos: densidade absoluta, densidade relativa, frequências absoluta e relativa, dominâncias absoluta e relativa e valor de importância; a diversidade específica foi dada pelo índice de diversidade de Shannon - Weaver (H') e pela equabilidade de Pielou (J').

\section{RESULTADOS}

No levantamento florístico, foram encontrados 1.491 indivíduos vivos, distribuídos em 36 espécies arbóreo-arbustivas lenhosas, 19 famílias e 31 gêneros.

As espécies encontradas foram Myracrodruon urundeuva Allemão, Schinopsis brasiliensis Engl, Spondias tuberosa Arruda/Anacardiaceae, Coudenbergia warmingiana Marchal ex. Warn./ Araliaceae, Ceiba glaziovii (Kuntze) K. Schum./ Bombacaceae, Varronia leucochephala (Moric.) J.S. Mill./Boraginaceae, Commiphora leptophloeos (Marth.) J.B. Gillett/Burseraceae, Bauhinia cheilantha (Bong.) Steud., Poincianella pyramidalis (Tul.) L. P. Queiroz, Senna macranthera (Collard) Irwin e Barneby/ Caesalpiniaceae, Cynophalla flexuosa (L.) J. Presl./ Capparaceae, Maytenus rigida Mart./Celastraceae, Croton rhamniflolius H.B.K., Croton blanchetianus Baill., Jatropha mollissima (Pohl) Baill., Manihot glaziovii Mull. Arg., Sapium glandulatum (Vell.) Pax./ Euphorbiaceae, Casearia sp/Flacourtiaceae, Byrsonima sp/Malpighiaceae, Senegalia bahiensis (Benth.) Seigler \& Ebinger, Senegalia piauhiensis (Benth.) Seigler \& Ebinger, Senegalias paniculata (Wild) Killip, Senegalia sp, Chloroleucon foliolosum (Benth.) G. P. Lewis, Mimosa ophthalmocentra Mart. ex. Benth, Mimosa tenuiflora (Wild.) Poir., Parapiptadenia zehntneri (Harms) M. P. Lima, Piptadenia stipulacea (Benth.) Ducke/Mimosaceae, Guapira noxia (Netto) Lundell/ Nyctaginaceae, Ruprechtia laxiflora Meisn./ Polygonaceae, Rhamnidium molle Reiss., Ziziphus joazeiro Mart./Rhamnaceae, Guettarda angelica Mart. ex Müll. Arg./Rubiaceae, Allophylus quercifolius (Mart.) Radlk/Sapindaceae, Aureliana fasciculata (Vell.) Sendtn/ Solanaceae e Helicteres ovata Lam/Sterculiaceae.

Revista Árvore, Viçosa-MG, v.36, n.5, p.851-858, 2012

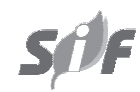


Quanto à riqueza de gêneros, os que se destacaram foram: Senegalia, com quatro espécies; Croton e Mimosa, com duas espécies; os demais 28 gêneros apresentaram uma única espécie. Foram encontradas 19 famílias no fragmento: Mimosaceae com nove espécies, Euphorbiaceae com cinco, Anacardiaceae e Caesalpiniaceae com três, Rhamnaceae com duas e as demais com apenas uma espécie. Assim, essas cinco famílias representaram um total de $61,11 \%$ do total registrado, enquanto 14 famílias responderam por apenas $38,89 \%$ do total de espécies.

Em relação às espécies arbóreas, pode-se destacar a Coudenbergia warmingiana/ Araliaceae (bordãode-velho), devido ao fato de esta não ter sido observada em outros levantamentos florísticos de caatinga.

Seis espécies foram consideradas raras no fragmento, em virtude da ocorrência de apenas um indivíduo na área avaliada, as arbóreas Commiphora leptophloeos, Guettarda angelica, Ruprechtia laxiflora, Senna macranthera e as arbustivas Senegalia sp e Helicteres ovata.

Outras cinco espécies arbóreas foram observadas na área estudada, porém, por não estarem inseridas nas parcelas, não foram amostradas essa ausência certamente foi em razão do pequeno número de indivíduos no fragmento. São elas: cumaru ou imburana-de-cheiro (Amburana cearensis (Allem.) A. C. Smith - Fabaceae), angico-de-caroço (Anadenanthera colubrina (Vell.) Brenan - Mimosaceae), jucá ou pau-ferro (Caesalpinia ferrea Mart. ex. Tul.- Caesalpiniaceae), uvaia (Eugenia sp-Myrtaceae) e canafístula (Senna spectabilis (DC.) H. S.Irwin \& Barneby-Caesalpiniaceae).

Quanto ao hábito de crescimento das espécies amostradas, o arbóreo predominou, com 29 espécies, sendo superior ao arbustivo, com apenas sete.

O índice de diversidade de Shannon-Weaver ( $\left.\mathrm{H}^{\prime}\right)$ foi de 2,05 nats/ind. e a equabilidade de Pielou, de 0,57 .

As estimativas dos parâmetros fitossociológicos das 10 espécies com as maiores densidades absolutas e relativas foram: Mimosa ophthalmocentra, Poincianella pyramidalis, Senegalia bahiensis, Senegalia paniculata, Croton blanchetianus, Jatropha mollissima, Piptadenia stipulacea, Mimosa tenuiflora, Aureliana fasciculata, Croton rhamniflolius; nas frequências absoluta e relativa foram as mesmas espécies, exceto Croton rhamniflolius, acrescida de Schinopsis brasiliensis. Com relação a dominância absoluta e relativa, foram as mesmas espécies, exceto Jatropha mollissima e Piptadenia stipulacea, acréscidas de Spondias tuberosa, Parapiptadenia zehntneri e Ceiba glaziovii; dessa maneira, as 10 espécies de maior valor de importância foram: Mimosa ophthalmocentra, Poincianella pyramidalis, Schinopsis brasiliensis, Senegalia bahiensis, Senegalia paniculata, Croton blanchetianus, Jatropha mollissima, Spondias tuberosa, Mimosa tenuiflora e Piptadenia stipulacea (Tabela 1).

As 10 espécies que apresentaram as maiores áreas basais foram, respectivamente: Mimosa ophthalmocentra, Poincianella pyramidalis, Spondias tuberosa, Schinopsis brasiliensis, Parapiptadenia zehntneri, Mimosa tenuiflora, Ceiba glaziovii, Senegalia bahiensis, Senegalia paniculata e Croton blanchetianus.

\section{DISCUSSÃO}

O número de espécies, famílias e gêneros encontrados no remanescente estava dentro da média dos levantamentos realizados nesse bioma. Os resultados, quanto ao número de espécies, famílias e gêneros, foram inferiores aos de Lemos e Rodal (2002), Pereira et al. (2002), Pegado et al. (2006), Lacerda et al. (2007), Rodal et al. (2008a), Santos et al. (2008) e Ramalho et al. (2009); sendo semelhantes aos encontrados por Lacerda et al. (2005) e superiores aos obtidos por Andrade et al. (2005), Queiroz et al. (2006), Pegado et al. (2006), Santana e Souto (2006), Fabricante e Andrade (2007), Pinheiro e Alves (2007), Pessoa et al. (2008), Rodal et al. (2008b) e Souza e Rodal (2010).

Segundo levantamento literário realizado por Lacerda et al. (2007), o número de espécies inventariadas na caatinga variou de 5 a 96. Dessa forma, o total de espécies arbóreo-arbustivas amostradas (36) foi expressivo.

Lacerda et al. (2007), avaliando o componente arbustivo-arbóreo de matas ciliares na bacia do rio Taperoá no semiárido paraibano, observaram que as famílias que apresentaram o maior número de espécies foram Mimosaceae (9), Euphorbiaceae (7) e Caesalpiniaceae (4), o que está de acordo com diversos autores que têm citado essas famílias como mais representativas em vários levantamentos da flora arbóreo-arbustiva realizados em área de caatinga (PEREIRA et al., 2002; LEMOS; RODAL, 2002;

Revista Árvore, Viçosa-MG, v.36, n.5, p.851-858, 2012 
Tabela 1 - Ordenação das espécies amostradas no fragmento de caatinga em Arcoverde, PE, de acordo com o VI, em que $\mathrm{N}=$ número de indivíduos; $\mathrm{AB}=$ Aréa Basal; $\mathrm{DA}=$ Densidade Absoluta; $\mathrm{DR}=$ Densidade Relativa; FA = Frequencia Absoluta; FR = Frequência Relativa; DoA = Dominância Absoluta; DoR = Dominancia Relativa; e VI = Valor de Importância.

Table 1 - Ordination of the species in the caatinga fragment in Arcoverde-PE according to the VI, where $N=n u m b e r$ of individuals; $A B=$ basal area, $D A=$ Absolute Density, $D R=$ Relative Density, $F A=$ Absolute Frequency, FR $=$ Relative Frequency, DoA = Absolute Dominance, DoR $=$ Relative Dominance e VI = Importance Value.

\begin{tabular}{|c|c|c|c|c|c|c|c|c|c|c|}
\hline Espécies & $\mathrm{N}$ & $\mathrm{ABm}^{2} / \mathrm{ha}$ & DAInd/ha & $\mathrm{DR}(\%)$ & $\mathrm{FA}(\%)$ & $\mathrm{FR}(\%)$ & $\mathrm{DoAm}^{2}$ & $\operatorname{DoR}(\%)$ & VI & $\mathrm{VI}(\%)$ \\
\hline Mimosa ophthalmocentra & 649 & 3,00 & 649 & 43,53 & 100 & 13,99 & 3,00 & 30,12 & 87,63 & 29,21 \\
\hline Poincianella pyramidalis & 436 & 3,66 & 436 & 29,24 & 100 & 13,99 & 3,66 & 36,72 & 79,95 & 26,65 \\
\hline Schinospis brasiliensis & 29 & 0,72 & 29 & 1,95 & 47,5 & 6,64 & 0,73 & 7,27 & 15,86 & 5,29 \\
\hline Senegalia bahiensis & 73 & 0,15 & 73 & 4,90 & 67,5 & 9,44 & 0,15 & 1,48 & 15,82 & 5,27 \\
\hline Senegalia paniculata & 63 & 0,12 & 63 & 4,23 & 60 & 8,39 & 0,12 & 1,17 & 13,78 & 4,59 \\
\hline Croton blanchetianus & 60 & 0,09 & 60 & 4,02 & 60 & 8,39 & 0,09 & 0,90 & 13,32 & 4,44 \\
\hline Jatropha mollissima & 41 & 0,06 & 41 & 2,75 & 50 & 6,99 & 0,06 & 0,58 & 10,32 & 3,44 \\
\hline Spondias tuberosa & 4 & 0,74 & 4 & 0,27 & 7,5 & 1,05 & 0,74 & 7,41 & 8,73 & 2,91 \\
\hline Mimosa tenuiflora & 18 & 0,28 & 18 & 1,21 & 17,5 & 2,45 & 0,28 & 2,78 & 6,43 & 2,14 \\
\hline Piptadenia stipulacea & 19 & 0,08 & 19 & 1,27 & 30 & 4,20 & 0,08 & 0,8 & 6,27 & 2,09 \\
\hline Parapiptadenia zehntneri & 3 & 0,35 & 3 & 0,20 & 7,5 & 1,05 & 0,35 & 3,55 & 4,80 & 1,60 \\
\hline Aureliana fasciculata & 10 & 0,01 & 10 & 0,67 & 17,5 & 2,45 & 0,01 & 0,13 & 3,24 & 1,08 \\
\hline Cynophalla flexuosa & 7 & 0,06 & 7 & 0,47 & 15 & 2,1 & 0,06 & 0,60 & 3,17 & 1,06 \\
\hline Ceiba glaziovii & 3 & 0,18 & 3 & 0,20 & 7,5 & 1,05 & 0,18 & 1,77 & 3,02 & 1,01 \\
\hline Sapium glandulatum & 8 & 0,03 & 8 & 0,54 & 15 & 2,10 & 0,03 & 0,31 & 2,94 & 0,98 \\
\hline Varronia leucochephala & 7 & 0,01 & 7 & 0,47 & 15 & 2,10 & 0,01 & 0,09 & 2,66 & 0,89 \\
\hline Croton rhamniflolius & 10 & 0,07 & 10 & 0,67 & 7,5 & 1,05 & 0,07 & 0,72 & 2,44 & 0,81 \\
\hline Chloroleucon foliolosum & 4 & 0,08 & 4 & 0,27 & 7,5 & 1,05 & 0,08 & 0,82 & 2,14 & 0,71 \\
\hline Rhamnidium molle & 4 & 0,01 & 4 & 0,27 & 10 & 1,40 & 0,01 & 0,14 & 1,81 & 0,60 \\
\hline Manihot glaziovii & 6 & 0,03 & 6 & 0,40 & 7,5 & 1,05 & 0,03 & 0,29 & 1,74 & 0,58 \\
\hline Senegalia piauhiensis & 4 & 0,03 & 4 & 0,27 & 7,5 & 1,05 & 0,03 & 0,34 & 1,66 & 0,55 \\
\hline Myracrodruon urundeuva & 2 & 0,06 & 2 & 0,13 & 5 & 0,70 & 0,06 & 0,64 & 1,47 & 0,49 \\
\hline Maytenus rigida & 3 & 0,01 & 3 & 0,20 & 7,5 & 1,05 & 0,01 & 0,12 & 1,37 & 0,46 \\
\hline Bauhinia cheilantha & 3 & 0,01 & 3 & 0,20 & 7,5 & 1,05 & 0,01 & 0,08 & 1,33 & 0,44 \\
\hline Ziziphus joazeiro & 3 & 0,04 & 3 & 0,20 & 5 & 0,70 & 0,04 & 0,4 & 1,30 & 0,43 \\
\hline Byrsonima sp & 3 & 0,01 & 3 & 0,20 & 5 & 0,70 & 0,01 & 0,07 & 0,97 & 0,32 \\
\hline Coudenbergia warmingiana & 5 & 0,02 & 5 & 0,34 & 2,5 & 0,35 & 0,02 & 0,24 & 0,93 & 0,31 \\
\hline Guapira noxia & 2 & 0,01 & 2 & 0,13 & 5 & 0,70 & 0,01 & 0,08 & 0,92 & 0,31 \\
\hline Casearia $\mathrm{sp}$ & 4 & 0,02 & 4 & 0,27 & 2,5 & 0,35 & 0,02 & 0,16 & 0,78 & 0,26 \\
\hline Allophylus quercifolius & 2 & 0,01 & 2 & 0,13 & 2,5 & 0,35 & 0,01 & 0,14 & 0,62 & 0,21 \\
\hline Guettarda angelica & 1 & 0,00 & 1 & 0,07 & 2,5 & 0,35 & 0,00 & 0,02 & 0,44 & 0,15 \\
\hline Ruprechtia laxiflora & 1 & 0,00 & 1 & 0,07 & 2,5 & 0,35 & 0,00 & 0,03 & 0,44 & 0,15 \\
\hline Senna macranthera & 1 & 0,00 & 1 & 0,07 & 2,5 & 0,35 & 0,00 & 0,01 & 0,43 & 0,14 \\
\hline Helicteres ovata & 1 & 0,00 & 1 & 0,07 & 2,5 & 0,35 & 0,00 & 0,01 & 0,43 & 0,14 \\
\hline Commiphora leptophloeos & 1 & 0,00 & 1 & 0,07 & 2,5 & 0,35 & 0,00 & 0,01 & 0,43 & 0,14 \\
\hline Senegalia sp & 1 & 0,00 & 1 & 0,07 & 2,5 & 0,35 & 0,00 & 0,01 & 0,43 & 0,14 \\
\hline
\end{tabular}

ANDRADE et al., 2005; LACERDA et al., 2005; SANTANA; SOUTO, 2006; PINHEIRO; ALVES, 2007; SOUZA; RODAL, 2010).

Quanto ao hábito de crescimento, os resultados indicaram que a área estudada se enquadrava como caatinga arbórea-arbustiva, com predominância das espécies arbóreas, sendo os dados semelhantes aos encontados por Ramalho et al. (2009) em uma área de caatinga, em Senhor do Bonfim, BA, com 28 espécies arbóreas, e superiores aos encontrados por Lemos e Rodal (2002) no Piauí, com 22 espécies arbóreas e 26 arbustivas; aos de Souza e Rodal (2010) em Floresta, PE, com 14 arbóreas e 11 espécies arbustivas; e inferiores aos encontrados por Lacerda et al. (2005) na Paraíba, com 44 espécies arbóreas e 18 arbustivas. 
Tabela 2 - Índice de diversidade de Shannon-Weaver (H') e equabilidade de Pielou (J'), encontrados neste estudo e em outros ambientes de caatinga.

Table 2 - Diversity index of Shannon-Weaver $\left(H^{\prime}\right)$ and equability of found in this work.

\begin{tabular}{llll}
\hline Autor/local & Local & H', \\
\hline Este estudo & Arcoverde-PE & 2,05 & 0,57 \\
Pereira et al. (2002) & Paraíba & 2,99 & 0,74 \\
Lemos e Rodal (2002) & Piauí & 3,00 & 0,74 \\
Alcoforado-Filho et al. (2003) & Pernambuco & 3,09 & 0,67 \\
Maracajá et al. (2003) & Rio Grande do Norte & 1,28 e 0,78 & 0,48 e 0,40 \\
Cestaro e Soares (2004) & Rio Grande do Norte & 3,19 e 3,26 & 0,79 e 0,86 \\
Andrade et al. (2005) & Paraíba & 1,51 e 1,43 \\
Santana e Souto (2006) & Rio Grande do Norte & 2,35 & 0,55 e 0,79 \\
Pegado et al. (2006) & Paraíba & 2,81 e 0,61 & 1,96 \\
Fabricante e Andrade (2007) & Paraíba & 1,10 e 0,86 \\
Pessoa et al. (2008) & Rio Grande do Norte & 2,49 \\
Santos et al. (2008) & Minas Gerais (Norte) & 0,79 e 0,22 \\
\hline
\end{tabular}

O índice de diversidade de Shannon-Weaver e a equabilidade de Pielou foram comparados com os de outros trabalhos em área de caatinga e demonstraram que a diversidade estava próxima da média apresentada em outros ensaios (Tabela 2), sendo superior à encontrada por Maracajá et al. (2003) e Andrade et al. (2005) nas duas áreas avaliadas, ao de Pegado et al. (2006) na área de caatinga invadida por Prosopis juliflora e superior à de Fabricante e Andrade (2007) e ao de Pessoa et al. (2008). A vegetação do fragmento avaliado apresentava maior riqueza de espécies quando comparada com a dessas áreas, o que pode ter sido decorrente do forte grau de antropização desses ambientes e das condições edafoclimáticas da área.

Para os sete parâmetros fitossociólogicos avaliados, as espécies Mimosa ophthalmocentra, Poincianella pyramidalis, Senegalia bahiensis, Senegalia paniculata, Croton blanchetianus e Mimosa tenuiflora sempre estiveram presentes entre as 10 espécies com os maiores valores, apesar do revezamento de posições entre elas nas parcelas.

Espécies como Spondias tuberosa, Parapiptadenia zehntneri e Ceiba glaziovii, apesar de apresentarem poucos indivíduos, obtiveram bom resultado em relação à dominância, em razão de possuírem grandes diamêtros, gerando, assim, maiores valores basais.

Mimosa ophthalmocentra e Poincianella pyramidalis foram as únicas espécies que tiveram $100 \%$ de frequência absoluta e valor de importância superior a $20 \%$, o que demonstra a dominância delas na população do fragmento estudado. A elevada distribuição de indivíduos dessas

Revista Árvore, Viçosa-MG, v.36, n.5, p.851-858, 2012 espécies na área significa que estas são as mais bem adaptadas ao ambiente avaliado, tanto nas condições edafoclimáticas quanto na competição com as demais espécies do fragmento de caatinga em Arcoverde, PE. Esse fato está de acordo com Sampaio (1996), que afirmou que Poincianella pyramidallis é a espécie que aparece com mais frequência no topo da maior parte das listas de estudos sobre a vegetação de caatinga, o que foi corroborado por Silva et al. (2012), que comentaram que a espécie é uma das que mostraram as maiores densidades e regeneração natural em área abandonada de caatinga.

\section{CONCLUSÃO}

O fragmento avaliado apresentava fisionomia arbóreoarbustivos com o predomínio de espécies arbóreas, boa diversidade e desenvolvimento fitofisionômico, e o número de espécies, famílias e gêneros amostrados estava dentro da média dos inventários fitossociológicos realizados nesse bioma. As espécies Mimosa ophthalmocentra e Poincianella pyramidalis foram as espécies mais bem adaptadas ao ambiente, pois foram as únicas que tiveram $100 \%$ de frequência absoluta e apresentaram valor de importância superior a $20 \%$.

\section{REFERÊNCIAS}

ALCOFORADO-FILHO, F. G.; SAMPAIO, E. V. S. B.; RODAL, M. J. N. Florística e fitossociologia de um remanescente de vegetação Caducifólia espinhosa arbórea em Caruaru, Pernambuco. Acta Botânica

Brasílica, v. 17, n.2, p.287-303, 2003. 
ALVES, J. J. A. Geoecologia da caatinga no semiárido do nordeste brasileiro. Climatologia e Estudos da Paisagem, v.2, n.1, p.58-71, 2007.

ANDRADE-LIMA, D. The caatingas dominium. Revista Brasileira de Botânica, v.4, n.2, p.149-153, 1981

ANDRADE, L. A. et al. Análise da cobertura de duas fitofisionomias de caatinga, com diferentes históricos de uso, no município de São João do Cariri, estado da Paraíba. Cerne, v.11, n.3, p.253-262, 2005.

BRASIL. Desertificação: caracterização e impactos. Brasília: Ministério do Meio Ambiente, dos Recursos Hídricos e da Amazônia Legal, 1999. 8p. (PROJETO PNUB/ BRA/93-36).

CAVALCANTI, L. C. B. Ipojuca: sua história, sua gente. Recife: Instituto de Coresponsabilidade pela Educação, 2010. 377p.

CESTARO; L. A.; SOARES, J. J. Variações florística e estrutural e relações fitogeográficas de um fragmento de floresta decídua no Rio Grande do Norte, Brasil. Acta Botânica Brasílica, v.18, n.2, p.203-208, 2004.

CIENTEC. Sistema para análise fitossociológica e elaboração dos planos de manejo de florestas nativas. Viçosa, MG: 2006. 295p.

CRONQUIST, A. The evolution and classification of flowering plants. New York: The New York Botanical Garden, 1988. 55p.

FABRICANTE, J. R.; ANDRADE, L. A. Análise estrutural de um remanescente de Caatinga no seridó paraibano. Oecologia Brasiliensis, v.11, n.3, p.341-349, 2007.

INSTITUTO BRASILEIRO DE GEOGRAFIA E ESTATISTICA - IBGE. Manual técnico da vegetação brasileira. Rio de Janeiro:

Departamento de Recursos Naturais e Estudos Ambientais, 1991. 92p. (Série Manuais Técnicos em Geociências, 1)

INSTITUTO BRASILEIRO DE GEOGRAFIA E EStATISTICA - IBGE. Censo do Município de Arcoverde-PE. Arcoverde: 2008. 8p.
LACERDA, A. V. et al. Estudo do componente arbustivo-arbóreo de matas ciliares na bacia do rio Taperoá, semi-árido paraibano: uma perspectiva para a sustentabilidade dos recursos naturais Oecologia Brasiliensis, v.11, n.3, p.331-340, 2007.

LACERDA, A. V. et al. Levantamento florístico do componente arbustivo-arbóreo da vegetação ciliar na bacia do rio Taperoá, PB, Brasil. Acta

Botânica Brasílica, v.19, n.3, p.615-623, 2005.

LEMOS J. R.; RODAL, M. J. N. Fitossociologia do componente lenhoso de um trecho da vegetação de caatinga no Parque Nacional Serra da Capivara, Piauí, Brasil. Acta Botânica Brasílica, v.16, n.1, p.23-42, 2002.

MARACAJÁ, P. B.; BATISTA, C. H. F.; SOUSA, A. H. Levantamento florístico e fitossociológico do estrato arbustivo-arbóreo de dois ambientes na Vila Santa Catarina, Serra do Mel, RN. Revista de Biologia e Ciências da Terra, v.3, n.2, p.25-32, 2003.

MARANGON, L. C. et al. Regeneração natural em um fragmento de floresta estacional semidecidual em Viçosa, Minas Gerais. Revista Árvore, v.32, n.1, p.183-191, 2008.

PEGADO, C. M. A. et al. Efeitos da invasão biológica de algaroba - Prosopis juliflora ( $\mathrm{Sw}$.) DC. sobre a composição e a estrutura do estrato arbustivo-arbóreo da caatinga no Município de Monteiro, PB, Brasil. Acta Botânica Brasílica, v.4, n.20, p.887-898, 2006.

PEREIRA, I. M. et al. Composição florística e análise fitossociológica do componente arbustivoarbóreo de um remanescente florestal no Agreste Paraibano. Acta Botânica Brasilica, v.16, n.3, p.357-369, 2002.

PESSOA, M. F. et al. Estudo da cobertura vegetal em ambientes da caatinga com diferentes formas de manejo no assentamento Moacir Lucena, Apodi RN. Revista Caatinga, v.21, n.3, p.40- 48, 2008.

PINHEIRO, K.; ALVES, M. Espécies arbóreas de uma área de Caatinga no sertão de Pernambuco, Brasil: dados preliminares. Revista Brasileira de Biociências, v.5, p.426-428, 2007.

Revista Árvore, Viçosa-MG, v.36, n.5, p.851-858, 2012 
QUEIROZ, J. A. et al. Análise da estrutura fitossociológica da Serra do Monte, Boqueirão, Paraíba. Revista de Biologia e Ciências da Terra, v.6, n.1, p.251-259, 2006.

RAMALHO, C. I. et al. Flora arbóreo-arbustiva em áreas de Caatinga no semi-árido baiano, Brasil. Revista Caatinga, v.22, n.3, p.182-190, 2009.

RODAL, M. J. N.; MARTINS, F. R.; SAMPAIO, E. V. S. B. Levantamento quantitativo das plantas lenhosas em trechos de vegetação de caatinga em Pernambuco. Revista Caatinga, v.21, n.3, p.192-205, 2008a.

RODAL, M. J. N.; COSTA, K. C. C.; LINS E SILVA, A. C. B. Estrutura da vegetação caducifólia espinhosa (Caatinga) de uma área do sertão central de Pernambuco. Hoehnea, v.35, n.2, p.209-217, 2008b.

SÁ, I. B.; RICHÉ, G. R.; FOTIUS, G. A. Fatores abióticos: áreas e ações prioritárias para conservação da caatinga. In: SILVA, J. M. C. et al. (Org.). Biodiversidade da caatinga: e ações prioritárias para conservação. Brasília: 2003. p.37-44.

SAMPAIO, E. V. S. B. Overview of the Brazilian Caatinga. In.: BULLOCK, S. H.; MOONEY, H. A.; MEDINA, E. Seasonally dry tropical forests. Cambrige: University Press, 1995. p.35-58.

SAMPAIO, E. V. S. B. Fitossociologia. In: SAMPAIO, E. V. S. B.; MAYO, S. J.; BARBOSA, M. R. V. (Eds.). Pesquisa botânica nordestina: progressos e perspectivas. Recife: Sociedade Botânica do Brasil/Seção Regional de Pernambuco. 1996. p. 203-230.
SANTANA, J. A. S.; SOUTO, J. S. Diversidade e Estrutura Fitossociológica da Caatinga na Estação Ecológica do Seridó-RN. Revista de Biologia e Ciências da Terra, v.6, n.2, p.232-242, 2006.

SANTOS, R. M. S. et al. Estrutura e florística de um remanescente florestal na fazenda Ribeirão, município de Juvenília, MG, Brasil. Revista Caatinga, v.21, n.4, p.154-162, 2008.

SILVA, J. M. C.; TABARELLI, M. Diversidade a adversidade. Recife: Universidade Federal de Pernambuco, 1999. p.7-11. (Cadernos de Extensão).

SILVA, S. O. et al. Regeneração natural em um remanescente de caatinga com diferentes históricos de uso no agreste pernambucano. Revista Árvore, v.36, n.3, p.441-450, 2012.

SOUZA, J. A. N.; RODAL, M. J. N.

Levantamento florístico em trecho de vegetação ripária de caatinga no Rio Pajeú, Floresta / Pernambuco - Brasil. Revista Caatinga, v.23, n.4, p.54-62, 2010.

TABArelli, M. et al. Análise de representatividade das unidades de conservação de uso direto e indireto na caatinga: análise preliminar. In: SILVA, J.M.C.; TABARELLI, M. (Coord.) WORKSHOP AVALIAÇÃO E IDENTIFICAÇÃO DE AÇÕES PRIORITÁRIAS PARA CONSERVAÇÃO, UTILIZAÇÃO SUSTENTÁVEL E REPARTIÇÃO DE BENEFÍCIOS DA BIODIVERSIDADE DO BIOMA CAATINGA. Petrolina: 2000. 